\title{
Gene Network Analysis During Early Infection of Human Coronary Artery Smooth Muscle Cells by Trypanosoma cruzi and Its gp83 Ligand
}

\author{
Pius N. Nde ${ }^{1}$ ), Candice A. Johnson ${ }^{1)}$, Siddharth Pratap ${ }^{1)}$, Tatiana C. Cardenas, Yuliya Y. \\ Kleshchenko, Vyacheslav A. Furtak, Kaneatra J. Simmons, Maria F. Lima, and Fernando \\ Villalta* \\ Department of Microbiology and Immunology, School of Medicine, Meharry Medical College, \\ 1005 Dr. D. B. Todd Jr. Blvd., Nashville, TN 37208-3599, USA
}

\begin{abstract}
Trypanosoma cruzi, the causative agent of Chagas' disease, infects heart and muscle cells leading to cardiac arrest followed by death. The genetic architectures in the early $T$. cruzi infection process of human cells are unknown. To understand the genetic architectures of the early invasion process of $T$. cruzi, we conducted gene transcription microarray analysis followed by gene network construction of the host cell response in primary human coronary artery smooth muscle (HCASM) cells infected with $T$. cruzi or exposed to T. cruzi gp83, a ligand used by the trypanosome to bind host cells. Using seven RT-PCR verified up-regulated genes (FOSB, ATF5, INPP1, CCND2, $T H B S 1, L A M C 1$, and APLP2) as the seed for network construction, we built an interaction network of the early $T$. cruzi infection process containing 165 genes, connected by 598 biological interactions. This interactome network is centered on the BCL6 gene as a hub. Silencing the expression of two seed genes (THBS1 and $L A M C 1$ ) by RNAi reduced T. cruzi infection. Overall, our results elucidate the significant and complex process involved in T. cruzi infection of HCASM cells at the transcriptome level. This is the first elucidation into the interactome network in human cells caused by $T$. cruzi and its gp 83 ligand.
\end{abstract}

\section{Keywords}

Gene network analysis; Gene transcription microarray analysis; Trypanosoma cruzi; Human coronary artery smooth muscle cells

\section{Introduction}

Trypanosoma cruzi, the protozoan parasite that causes Chagas' disease, is an obligate intracellular parasite that infects heart and muscle cells among others leading to cardiac arrest followed by death. The World Health Organization estimates that almost one-quarter of the Latin American population is permanently at risk, with over 15 million people infected with T. cruzi [1]. Chagas' disease is now viewed as an emerging illness associated with human immunodeficiency virus type 1 infection, where it induces dramatic brain pathology and early death [2]. Another serious health problem is the incidence of human-tohuman transmission through exposure to blood contaminated with trypomastigotes during pregnancy or blood transfusion [3]. The latter has been an issue of great concern in the

(phone: (615) 327-6667; fax: (615) 327-6072; fvillalta@ mmc.edu).

1) P. N. N., C. A. J., and S. P. contributed equally to this work. 
United States due to the millions of Latin American immigrants in the United States [4]. The American Red Cross has only recently begun to screen for T. cruzi in the U.S. donor blood supply [5]. Currently, there is no cure for chronic Chagas' disease. Further, the drugs available for the acute stage of infection are highly toxic to humans [6]. The development of an efficacious prophylactic vaccine faces many challenges, and progress is slow despite global health efforts. The disease causes significant morbidity and mortality in the Americas, yet it remains incurable. Despite the magnitude of this public health problem, almost nothing is known about the interactome networks in the process of $T$. cruzi infection, which is critically important to the understanding of the molecular pathogenesis of $T$. cruzi infection [7][8] and developing novel approaches of intervention in Chagas'disease. The interaction between the invasive trypomastigote form of the parasite and host cell receptors is the first critical step during the initial process of infection [7][8]. Our group and others have employed gene transcript microarray technology and have shown that certain host genes are significantly modulated by the parasite to facilitate the process of infection [9-14]. An integrative analysis of the observed gene expression profile at the transcriptome level as well as protein-protein interaction (PPI) networks can provide insights into the molecular mechanisms of Chagas' disease. Very recently, layered PPI networks have been used to provide unique insights into the mechanism of dilated cardiomyopathy [15]. PPI network analysis has thus far not been used to elucidate the complex biological interactions during the initial phases of $T$. cruzi infection. Here we studied the gene expression profile in human coronary artery smooth muscle (HCASM) cells modulated by $T$. cruzi trypomastigotes and T. cruzi gp83 ligand [16-18] and report the interactome network operating in HCASM cells during the early process of $T$. cruzi invasion. This is the first elucidation into the interactome network in human cells induced by $T$. cruzi and its gp 83 ligand.

\section{Results and Discussion}

\section{Validation of Microarray Analysis and Kinetics of Cellular Gene Expression in HCASMC Induced by T. cruzi and T. cruzi gp83}

Invasive $T$. cruzi trypomastigotes have been shown to modulate the gene expression profile of host cells in order to facilitate their invasion and cellular establishment with subsequent development of pathology. To unravel the gene profile modulated by the parasites, we exposed HCASM cells to invasive T. cruzi trypomastigotes or its gp83 ligand and determined the changes in the gene expression profile induced by the parasite and its ligand by gene expression microarray analysis. We observed that several gene transcripts were modulated by the parasite and its gp 83 ligand. The microarray data was validated by quantitative real time-PCR (RT-PCR) using seven of the T. cruzi trypomastigote upregulated genes (INPP1, FOSB, CCND2, LAMC1, THBS1, APLP2 and ATF5) and by the $T$. cruzi trypomastigote gp83 ligand (INPP1 and LAMC1). Indeed, the levels of expression of all of these genes induced by $T$. cruzi or its gp 83 were verified by RT-PCR as indicated in Fig. 1, which shows the kinetics of gene expression in HCASM cells induced by $T$. cruzi. Further, T. cruzi gp83 induces the same kinetics of expression of INPPI and LAMCl in HCASM cells as whole parasites as determined by RT-PCR. The effect induced by gp 83 and the whole trypanosome on the INPPI and LAMCI expression in HCASM cells is consistent with the fact that $T$. cruzi $\mathrm{gp} 83$ has two forms, a GPI membrane anchored molecule and a PLC-cleaved molecule released into the medium. This is also consistent with previous reports from our group showing that the recombinant gp83 is able to activate the MAP kinase pathway in human cells to promote parasite entry [17]. The parasite up-regulated the expression of the transcription factors FOSB and ATF5. The kinetics of FOSB expression induced by $T$. cruzi in HCASM cells indicates that $F O S B$ was significantly increased (more than eight-fold) at $120 \mathrm{~min}$ and then decreased at $180 \mathrm{~min}$. ATF5 showed a gradual increase at $60 \mathrm{~min}$ which decreased to normal levels at $180 \mathrm{~min}$. Others have also shown that $T$. cruzi 
is able to activate transcription factors that widely modulate the mRNA levels of the host cells [11][13]. FOSB and ATF5 mRNA levels are increased by exposure of HCASM cells to the parasite. These transcription factors are a part of the Activator Protein (AP-1) complex, and therefore, may initiate the modulation of the host cell transcriptome in favor of parasite entry, establishment of infection and the development of pathology. Activation of AP-1 induces downstream activation of endothelin, which is involved in the pathogenesis of chagasic cardiomyopathy [19].

The parasite and its gp83 ligand significantly increased expression of the signal transduction protein INPPI in order to facilitate cellular invasion. We also observe that the parasite induces an increase in the expression of Cyclin D2 indicating that the infected HCASM cells may have a disregulation of their proliferative status. The parasite significantly increased (14-fold at $120 \mathrm{~min}$ ) the expression of LAMCl (laminin $\gamma$-1), which is a major component of the extracellular matrix, and THSB- 1 (thrombospondin-1), which is a matricellular glycoprotein. THSB-1 transcript levels increase approximately six-fold at $60 \mathrm{~min}$, followed by an increase of 2.7 -fold at 120 min during infection of HCASM cells by $T$. cruzi. The transcript levels of $T H S B-2, T H S B-3$ and $T H S B-4$ do not change significantly during the same period of $T$. cruzi infection of HCASM cells. Increased expression of these extracellular matrix proteins may cause the cardiac remodeling observed in Chagas' disease.

\section{Gene Network Triggered by T. cruzi and T. cruzi gp83}

The modulation of host gene expression patterns by invasive $T$. cruzi trypomastigotes and/or its gp83 ligand lead to changes in the Protein-Protein-Interaction (PPI) network patterns of the cells (Figs. 2 - 8). The identification and unraveling of the complex PPI networks at the molecular and cellular levels will enhance our understanding of the initiation and development of Chagas' disease. In order to understand the relevant biological networks induced in HCASM cells by T. cruzi, we used RT-PCR validated genes from the microarray analysis in order to generate the PPI network shown in Fig. 9.

The interaction network of $T$. cruzi infection of HCASM cells was seeded with the seven RT-PCR verified transcripts as initial nodes. The network was expanded to one degree of direct interaction using the MiMI pluggin for cytoscape [20]; resulting in seven seednetworks with the seed node as a center point. The seven seed-networks were merged for common interactions and connections. The resulting interaction network contains 165 nodes representing biological entities (genes/transcripts/proteins), connected by 598 edges representing biological interactions/associations (Fig. 9). The sub-networks of Cyclin D2 $(C C D N-2)$ and $F B J$ murine osteosarcoma viral oncogene homolog B (FOSB) share the most common inter-connections while the sub-network of activating transcription factor 5 $(A T F-5)$ is the most distant from the others (Fig. 9). Interestingly, B-cell lymphoma 6 $(B C L 6)$ is a central hub of the interaction network, common to five of the seven subnetworks by one degree of connection to the initial seed nodes.

2.1. FOSB Sub-Network (Fig. 2)—FBJ murine osteosarcoma viral oncogene homolog B (FOSB) transcription factor is part of the FOS gene family. The two genes encode a leucine zipper, which can dimerize with the Jun family of proto-oncogenes and activate AP-1 complex. Activation of AP-1 controls different cellular functions including proliferation, differentiation and transformation. As shown in Fig. 2, FOSB interacts with JUNB, SMADs, $A T F s$, and $T A F$ to facilitate interaction with $B C L 6$. The transcription suppressor $B C L 6$ suppresses the cells defensive chemistry thereby facilitating the process of cellular invasion by $T$. cruzi. Repression of AP-1 function by BCL6 may be a key mechanism through which $B C L 6$ may regulate gene expression to control inflammation and differentiation in Chagas' disease [21]. 
2.2. ATF5 Sub-Network (Fig. 3)—ATF5, a member of the activating transcription/cAMP response element binding protein $(A T F / C R E B)$ family, is a $b Z I P$ transcription factor [22], which can interact with a variety of proteins including Cyclin D3 [23]. Modulation of ATF5 expression affects its interaction with $E 2 F 1$ and cyclin D3 (CCND3) preventing apoptosis and enhancing parasite survival [24]. AFT5 promotes cell survival and increases tolerance of cardiomyocytes through transcriptional activation of HSP27 [25]. The anti-apoptotic role of ATF5 [26][27] is critical for continued parasite survival in infected HCASM cells.

Furthermore, the PPI network output shows that ATF5 also interacts with other transcription factors that regulate cellular inflammatory responses thereby enhancing parasite survival in infected HCASM cells (Fig. 3).

2.3. INPP1 Sub-Network (Fig. 4)—Increase in the cellular concentration of inositol phosphatases would increase inositol turnover of parasitized HCASM cells. INPPI plays an important role in cell signaling via the PI3-kinase pathway. Cellular up-regulation of PI3 phosphatases and consequently other PI3-kinase pathway proteins, leads to an increase in the activation of the cell signaling cascade. PI3-kinases are critical for many cellular processes important for $T$. cruzi infection including cytoskeletal rearrangement, membrane trafficking, and endosome fusion [28][29].

2.4. CCND2 Sub-Network (Fig. 5)—Cell cycle progression is regulated at multiple checkpoints; regulated in part by a family of protein kinases (cyclin-dependent kinases or $C D K s$ ) and their activating partners (the cyclins). The parasite may induce expression of cyclin D2 (CCND2) to ensure that the infected HCASM cells can initiate the disregulation of the successive phases of the cell cycle.

Cyclin D2 is required for induction of adult cell cycle reentry [30] and its induction promotes proliferation but not hypertrophy of VSMCs. It was demonstrated that CCND2 expression peaks during the chronic stage of $T$. cruzi infection of mice, suggesting that adult mouse myocardial cells revert to a proliferative phenotype [31]. TGF- $\beta$ is also found to be up-regulated during the acute phase of $T$. cruzi infection [31]. TGF- $\beta, C C N D 2$, and PI3kinase signaling pathways have common interconnections. For example, periostin mediated mRNA expression in cultured VSMCs was stimulated by TGFß1 through the PI3-kinasedependent signaling pathway.

2.5. THBS1 Sub-Network (Fig. 6)—Thrombospondin 1 (THSB1) is a matricellular glycoprotein that inhibits angiogenesis and modulates endothelial cell adhesion, motility, and growth. THSB1 interacts with several cell adhesion receptors, including CD36, integrins, and integrin-associated proteins and inhibits matrix metalloproteinase enzymes thereby remodeling the cellular microenvironment [32]. We have shown that T. cruzi upregulates the expression of THSBI to facilitate the invasion process [10]. Knockdown of THSBI by RNAi significantly inhibited cellular invasion by $T$. cruzi (Fig. 10). The increased level of $T H S B 1$ expression significantly modulates the interactome cross talk between the cells, as we found the largest PPI sub-network obtained occurred in the THSB1 seed network, with 51 nodes interconnected with 151 edges (Fig. 6). This network topology will potentially favor parasite multiplication and survival. During this cross talk, higher levels of THSBI activate TGF- $\beta$ [33], a major pro-fibrotic cytokine causing modification of the ECM milieu. THSB 1 then interacts with several host proteins including the COL7A1 gene product, which then interacts with other proteins from the laminin family of genes to make the ECM conducive to parasite mobility and cellular invasion. Since the modulation of THSB1 gene networking profile is very critical for parasite mobility and cellular invasion, several parasites (including the human pathogens Plasmodium, Toxoplasma, and Cryptosporidium) that have not been shown to up-regulate human THSB1, use a transmembrane thrombospondin-related anonymous protein (TRAP) for gliding motility and invasion of 
vertebrate host cells [34-36]. TRAP proteins produced by the parasite can also activate TGF$\beta$, an anti-inflammatory cytokine that counteracts the effects of inflammatory cytokines like IL-12, INF- $\gamma$ and TNF- $\alpha$ thereby facilitating parasite survival.

Modulation of THSB1 expression in HCASM cells by $T$. cruzi is essential because THSB1 blocks the cytoprotective activity of nitric oxide (NO) by antagonizing the NO/cGMP signaling pathway thereby negatively regulating vascular tone, vascular smooth muscle cells adhesion, chemotaxis and proliferation [37].

Increased THSB1 transcripts in parasitized HCASM cells may suggest that THSB1 also contributes in part to the pathology caused by $T$. cruzi. Its up-regulation indicates that the parasite exploits THSB1 in addition to laminin to remodel the ECM and navigates through it to facilitate infection. THSBI is required for the infection process of $T$. cruzi as evidenced by RNAi of that specific isoform (Fig. 10). The up-regulation of host THSBI expression by $T$. cruzi to facilitate the infection of human cells represents a new mechanism that contributes to the pathogenesis of $T$. cruzi infection.

2.6. LAMC1 Sub-Network (Fig. 7)—Recent evidence indicates that T. cruzi modulates the extracellular matrix network to facilitate infection of human cells [9][10]. The T. cruzi gp83 ligand increases the level of laminin $\gamma-1$ (LAMC1) transcript and its expression in mammalian cells, leading to an increase in cellular infection. RNAi of host cell $L A M C 1$ knocks down the levels of its transcript (Fig. 10) as well as corresponding LAMC1 protein expression in mammalian cells, causing a dramatic reduction in cellular infection by $T$. cruzi [9][10]. Thus, host $L A M C 1$, which is regulated by the parasite, plays a crucial role in the early process of infection.

Previous studies have shown that parasite molecules bind to immobilized laminin [38] and that human galectin-3 enhances this interaction [39], suggesting that the trypanosome interacts with laminin and $L A M C 1$ is the most abundant isoform in humans [40]. The hypothesis that $T$. cruzi gp83 binds to human cells to regulate the expression of $L A M C 1$, which is required for $T$. cruzi infection, is experimentally supported by the fact that preexposure of HCASM cells to $T$. cruzi gp83 up-regulates T. cruzi infection and expression of $L A M C 1$, and that knocking down the expression of $L A M C 1$ by RNAi dramatically reduces T. cruzi infection of human cells (Fig. 10).

Host $L A M C 1$, which is regulated by the parasite ligand gp83, plays a crucial role in the early process of $T$. cruzi infection of heart cells. The regulation of gp83 expression by the parasite as well as the gp 83 released by the trypanosome PLC plays an important role in the modulation of early signaling events leading to initial parasite infection. The regulation of infection by the gp 83 ligand represents a parasite escape mechanism by which invasive trypomastigotes release gp83 to efficiently gain entry into human heart cells.

T. cruzi must navigate through the basal lamina, which contains $L A M C l$, and surrounds individual muscle cells such as HCASM cells before infecting these cells. The fact that gp83 increases $L A M C 1$ transcript levels in human coronary artery smooth muscle cells, correlates with the finding that laminin is deposited in the hearts of patients infected with Chagas' disease [41]. This suggests that the regulation of $L A M C 1$ in heart cells by gp83 might explain, in part, the cause of this pathology.

The fact that the T. cruzi gp83 ligand remodels the extracellular matrix (ECM) by upregulating the expression of $L A M C 1$, together with the report that $T$. cruzi presents laminin receptors on its surface [38] indicates that the parasite exploits $L A M C 1$ to navigate through the ECM to facilitate infection. Thus, the T. cruzi gp83 ligand is a virulence factor that 
modifies LAMC1 expression in the ECM to contribute to the pathogenesis of T. cruzi infection in human heart cells.

2.7. APLP2 Sub-Network (Fig. 8)—Amyloid beta (A4) precursor-like protein 2 (APLP2) associates with antigen presentation molecules (like MHC class I molecules) and regulates their surface expression by enhancing endocytosis, which may be involved in the process of $T$. cruzi infection. Furthermore, APLP2 has been shown to interact with APBB1, which is associated with plaque formation in Alzheimer's disease. Amyloids are insoluble proteins with altered secondary structure which are abnormally deposited in organs and tissues. The structure of the deduced amino acid sequence of amyloid B (A4) suggests it is a glycosylated cell-surface receptor consisting of an $\mathrm{N}$-terminal signal sequence, three extracellular domains, a transmembrane region, and a cytoplasmic domain [42]. Amyloid B (A4) was found to be downregulated in T. cruzi infected HeLa cells in comparison to noninfected controls after three days of infection [12]. However, its level of expression during early infection was not previously investigated by this group. Moreover, serum amyloid $\mathrm{P}$ was up-regulated in chronic T. cruzi infected mice [31]. Interestingly, cell surface GPI mucins of $T$. cruzi induced expression of serum amyloid A3 protein in macrophages, which is an acute phase protein involved in the immune response to T. cruzi [43].

The global interaction network of $T$. cruzi infection of HCASM cells is depicted in Fig.9.

\section{Gene Function in T. cruzi Infection}

Silencing the expression of $L A M C 1$ and THSBI by stable RNAi significantly reduces $T$. cruzi infection in HeLa cells (Fig. 10). Panel A presents an immunoblot of LAMC1 RNAi showing a significant reduction in the expression in $L A M C 1$ in HeLa cells, whereas panel B presents an immunoblot showing that THBS1 RNAi reduces the expression of THBS1 in HeLa cells. Panel C shows the kinetics of T. cruzi infection in HeLa cells in which LAMC1 and THBS1 were stably silenced by RNAi, indicating that silencing of $L A M C 1$ and THBS1 genes dramatically reduces cellular infection by $T$. cruzi. These results indicate that host $L A M C 1$ and THBS1, which are regulated by the parasite, play critical roles in the early process of $T$. cruzi infection. Our results indicate that the T. cruzi gp83, which the parasite uses to attach to host cells, increases the expression of $L A M C 1$, leading to an increase in cellular infection and plays a crucial role in the early process of infection. Since the gp 83 is a membrane GPI-anchored molecule that is cleaved by the trypanosome PLC, this may represent a mechanism of escape by which the invasive trypomastigotes release gp 83 to efficiently gain entry into human cells [9]. Furthermore, the released trypanosome gp83 ligand is able to increase extracellular matrix $L A M C 1$ to recruit $T$. cruzi at the site of infection to promote entry. Thus, the T. cruzi gp83 ligand is a virulence factor that modifies $L A M C 1$ expression in the ECM to contribute to the pathogenesis of $T$. cruzi infection in human heart cells.

\section{Experimental Part}

\section{Parasite Cultures}

Pure highly invasive trypomastigote cultures (clone MMC 20A) derived from the Tulahuen strain of $T$. cruzi were obtained from the supernatant of heart myoblast monolayers as previously described [44].

\section{Infection assays}

Parasite binding to human cells was evaluated at $2 \mathrm{~h}$ by fluorescence microscopy with fluorescein isothiocyanate (FITC)-labeled antibodies to a trypomastigote surface protein and 4',6'-diamidino-2-phenylindole (DAPI) [9]. The number of bound, FITC-fluorescent 
parasites per 200 cells was determined. Parasite entry was evaluated at the same 2-h time point. The number of internalized parasites was obtained by subtracting the number of bound, FITC-fluorescent parasites from the total number of DAPI-stained kinetoplast DNA parasites per 200 host cells. Parasite multiplication within cells was evaluated at $72 \mathrm{~h}$ by standardized procedures [44]. Infection assays with primary human coronary artery smooth muscle (HCASM) cells and HeLa cells were done in triplicate, and experiments were repeated three times.

\section{Quantization of Host Gene Expression by Real-Time PCR}

First strand cDNA was synthesized from $5 \mu \mathrm{g}$ of high quality RNA using Superscript III as described by the manufacturer (Invitrogen, Grand Island, NY). The cDNAs were used for quantitative Real-Time PCR experiments to determine the transcript level as previously described [9]. The primers used for the Real-Time PCR experiments were designed with the Beacon Designer 4.0 program (Premier Biosoft, Palo Alto, CA). The primer sequences used are shown in the Table.

\section{cDNA Microarrays and Gene Analysis}

Monolayers of HCASM cells (Cambrex, Walkersville, MD) were washed with smooth muscle cell basal medium (SmBM) from Cambrex and incubated with either $T$. cruzi trypomastigotes (10 parasites per cell) or with recombinant $T$. cruzi gp83 free of endotoxin [9][6] in SmBM or with SmBM alone for 30, 60, 120, or $180 \mathrm{~min}$. Monolayers were solubilized with TriZol and RNA was purified as previously described [9].

\section{Gene Transcription Microarrays}

Gene transcription microarrays were done using custom human cDNA microarrays spotted on glass slides by the Vanderbilt Microarray Shared Resource. The clones were from Research Genetics Sequence Verified Library (ResGen Division of Invitrogen Corp., Carlsbad, CA) containing a panel of ten-thousand probes sets, derived from the IMAGE consortium collection. Two micrograms of mRNA were used in the microarray assays as described [45]. Relative expression levels of gene transcripts are the average of two replicates. Normalization was conducted by interquartile method and only transcripts having a relative expression change of 2-fold up or down regulated between normal and infected states were filtered through. Additionally a $t$-test was applied to the normal versus infected states and only genes with a $t$-test derived $p$-value of $<0.05$ and up or down-regulated 2 -fold or more, were considered as significantly changed for added stringency.

\section{Gene Interaction Network Construction}

In order to populate and build our interaction network, the MiMI cytoscape plugin (version 3.2) was used. MiMI retrieves molecular interactions from the Michigan Molecular Interactions (MiMI) database and displays the interaction network with cytoscape. MiMI gathers and merges data from well-known protein interaction databases including BIND, DIP, HPRD, RefSeq, SwissProt, IPI, and CCSB-HI1. A provenance model has been developed that tracks the source of each data element and what processes have been performed upon it. The Plugin also integrates with other NCIBI tools for literature information, document summarization and pathway matching [20]. The RT-PCR verified transcripts were used as the initial population nodes. MiMI was used to query for the initial nodes and their respective nearest neighbors to one degree. The networks were then merged for interconnections and the global interactome was visualized in cytoscape (version 2.6.1) using the yFiles: organic layout format [46]. 


\section{Acknowledgments}

This work was supported by NIH grant 5SC1 AI080580-03 to F. V.; C. A. J. and T. C. C. are pre-doctoral fellows supported by NIH grant 2T32AI007281-21. $P$. $N$. is supported by NIH grant 1SC2AI083925-01. M. F. L. is supported by NIH grant 5R25GM059994-10, and $S$. $P$. is supported by NIH grant 5G12RR003032-25. Experimental set-up, interpretation and data analysis were performed through the use of the Meharry Medical College Microarray and Bioinformatics Core (http://www.mmc.edu/bioinformatics/) supported by NIH grant 5R25GM059994-S1 and the Meharry Medical College Proteomics Core supported by NIH grant 1U54RR026140-0109.

\section{REFERENCES}

1. http://www.who.int/tdr/diseases/chagas/default.htm.

2. Rosemberg S, Chaves CJ, Higuchi ML, Lopes MBS, Castro LHM, Machado LR. Neurology 1992;42:640. [PubMed: 1549229]

3. Nickerson P, Orr P, Schroeder M-L, Sekla L, Johnston JB. Ann. Intern. Med 1989;111:851. [PubMed: 2510572]

4. Kirchhoff LV. Ann. Intern. Med 1989;111:773. [PubMed: 2510570]

5. McCarthy M. Lancet 2003;362:1988. [PubMed: 14686364]

6. Castro JA, de Mecca MM, Bartel LC. Hum. Exp. Toxicol 2006;25:471. [PubMed: 16937919]

7. Villalta F, Madison MN, Kleshchenko YY, Nde PN, Lima MF. Frontiers Biosci 2008;13:3714.

8. Villalta F, Scharfstein J, Ashton AW, Tyler KM, Guan F, Mukherjee S, Lima MF, Alvarez S, Weiss LM, Huang H, Machado FS, Tanowitz HB. Parasitol. Res 2009;104:1251. [PubMed: 19283409]

9. Nde PN, Simmons KJ, Kleshchenko YY, Pratap S, Lima MF, Villalta F. Infect. Immun 2006;74:1643. [PubMed: 16495535]

10. Simmons KJ, Nde PN, Kleshchenko YY, Lima MF, Villalta F. FEBS Lett 2006;580:2365. [PubMed: 16616140]

11. Vaena de Avalos S, Blader IJ, Fisher M, Boothroyd JC, Burleigh BA. J. Biol. Chem 2002;277:639. [PubMed: 11668183]

12. Shigihara T, Hashimoto M, Shindo N, Aoki T. Parasitol. Res 2008;102:715. [PubMed: 18058129]

13. Mukherjee S, Belbin TJ, Spray DC, Iacobas DA, Weiss LM, Kitsis RN, Wittner M, Jelicks LA, Scherer PE, Ding A, Tanowitz HB. Parasitol. Res 2003;91:187. [PubMed: 12910413]

14. Imai K, Mimori T, Kawai M, Koga H. Microbiol. Immunol 2005;49:623. [PubMed: 16034205]

15. Zhu W, Yang L, Du Z. PLoS One 2009;4:e6288. [PubMed: 19629191]

16. Villalta F, Smith CM, Ruiz-Ruano A, Lima MF. FEBS Lett 2001;505:383. [PubMed: 11576533]

17. Villalta F, Zhang Y, Bibb KE, Burns JM Jr, Lima MF. Biochem. Biophys. Res. Commun 1998;249:247. [PubMed: 9705866]

18. Villalta F, Lima MF, Ruiz-Ruano A, Zhou L. Biochem. Biophys. Res. Commun 1992;182:6. [PubMed: 1370617]

19. Huang H, Petkova SB, Cohen AW, Bouzahzah B, Chan J, Zhou JN, Factor SM, Weiss LM, Krishnamachary M, Mukherjee S, Wittner M, Kitsis RN, Pestell RG, Lisanti MP, Albanese C, Tanowitz HB. Infect. Immun 2003;71:2859. [PubMed: 12704159]

20. Gao J, Ade AS, Tarcea VG, Weymouth TE, Mirel BR, Jagadish HV, States DJ. Bioinformatics 2009;25:137. [PubMed: 18812364]

21. Vasanwala FH, Kusam S, Toney LM, Dent AL. J. Immunol 2002;169:1922. [PubMed: 12165517]

22. Lee KA, Hai TY, SivaRaman L, Thimmappaya B, Hurst HC, Jones NC, Green MR. Proc. Natl. Acad. Sci. U.S.A 1987;84:8355. [PubMed: 2960975]

23. Liu W, Sun M, Jiang J, Shen X, Sun Q, Liu W, Shen H, Gu J. Biochem. Biophys. Res. Commun 2004;321:954. [PubMed: 15358120]

24. Wei Y, Jiang J, Sun M, Chen X, Wang H, Gu J. Biochem. Biophys. Res. Commun 2006;339:591. [PubMed: 16300731]

25. Wang H, Lin G, Zhang Z. Cell Biol. Int 2007;31:1309. [PubMed: 17606386]

26. Persengiev SP, Devireddy LR, Green MR. Genes Devel 2002;16:1806. [PubMed: 12130540] 
27. Angelastro JM, Canoll PD, Kuo J, Weicker M, Costa A, Bruce JN, Greene LA. Oncogene 2006;25:907. [PubMed: 16170340]

28. Molkentin JD. Circulat. Res 2000;87:731. [PubMed: 11055975]

29. Passier R, Zeng H, Frey N, Naya FJ, Nicol RL, McKinsey TA, Overbeek P, Richardson JA, Grant SR, Olson EN. J. Clin. Invest 2000;105:1395. [PubMed: 10811847]

30. Zhong W, Mao S, Tobis S, Angelis E, Jordan MC, Roos KP, Fishbein MC, de Alborán IM, MacLellan WR. EMBO J 2006;25:3869. [PubMed: 16902412]

31. Mukherjee S, Nagajyothi F, Mukhopadhyay A, Machado FS, Belbin TJ, Campos de Carvalho A, Guan F, Albanese C, Jelicks LA, Lisanti MP, Silva JS, Spray DC, Weiss LM, Tanowitz HB. Genomics 2008;91:423. [PubMed: 18343633]

32. Sid B, Sartelet H, Bellon G, El Btaouri H, Rath G, Delorme N, Haye B, Martiny L. Crit. Rev. Oncol. Hematol 2004;49:245. [PubMed: 15036264]

33. Ribeiro SM, Poczatek M, Schultz-Cherry S, Villain M, Murphy-Ullrich JE. J. Biol. Chem 1999;274:13586. [PubMed: 10224129]

34. Kappe S, Bruderer T, Gantt S, Fujioka H, Nussenzweig V, Menard R. J. Cell Biol 1999;147:937. [PubMed: 10579715]

35. Sibley LD. Science 2004;304:248. [PubMed: 15073368]

36. Morahan BJ, Wang L, Coppel RL. Trends Parasitol 2009;25:77. [PubMed: 19101208]

37. Isenberg JS, Maxhimer JB, Hyodo F, Pendrak ML, Ridnour LA, DeGraff WG, Tsokos M, Wink DA, Roberts DD. Am. J. Pathol 2008;173:1100. [PubMed: 18787106]

38. Giordano R, Fouts DL, Tewari D, Colli W, Manning JE, Alves MJM. J. Biol. Chem 1999;274:3461. [PubMed: 9920891]

39. Moody TN, Ochieng J, Villalta F. FEBS Lett 2000;470:305. [PubMed: 10745086]

40. Sasaki T, Fässler R, Hohenester E. J. Cell. Biol 2004;164:959. [PubMed: 15037599]

41. Milei J, Sánchez J, Storino R, Yu Z-X, Denduchis B, Ferrans VJ. Mol. Cell. Biochem 1993;129:161. [PubMed: 8177238]

42. Lemaire HG, Salbaum JM, Multhaup G, Kang J, Bayney RM, Unterbeck A, Beyreuther K, MullerHill B. Nucleic Acids Res 1989;17:517-522. [PubMed: 2783775]

43. Ferreira LR, Silva AM, Michailowsky V, Reis LF, Gazzinelli RT. J. Leukocyte Biol 1999;66:593. [PubMed: 10534115]

44. Lima MF, Villalta F. Mol. Biochem. Parasitol 1989;33:159. [PubMed: 2657421]

45. http://vmsr.net/.

46. Cline MS, Smoot M, Cerami E, Kuchinsky A, Landys N, Workman C, Christmas R, AvilaCampilo I, Creech M, Gross B, Hanspers K, Isserlin R, Kelley R, Killcoyne S, Lotia S, Maere S, Morris J, Ono K, Pavlovic V, Pico AR, Vailaya A, Wang PL, Adler A, Conklin BR, Hood L, Kuiper M, Sander C, Schmulevich I, Schwikowski B, Warner GJ, Ideker T, Bader GD. Nat. Protocols 2007;2:2366. 


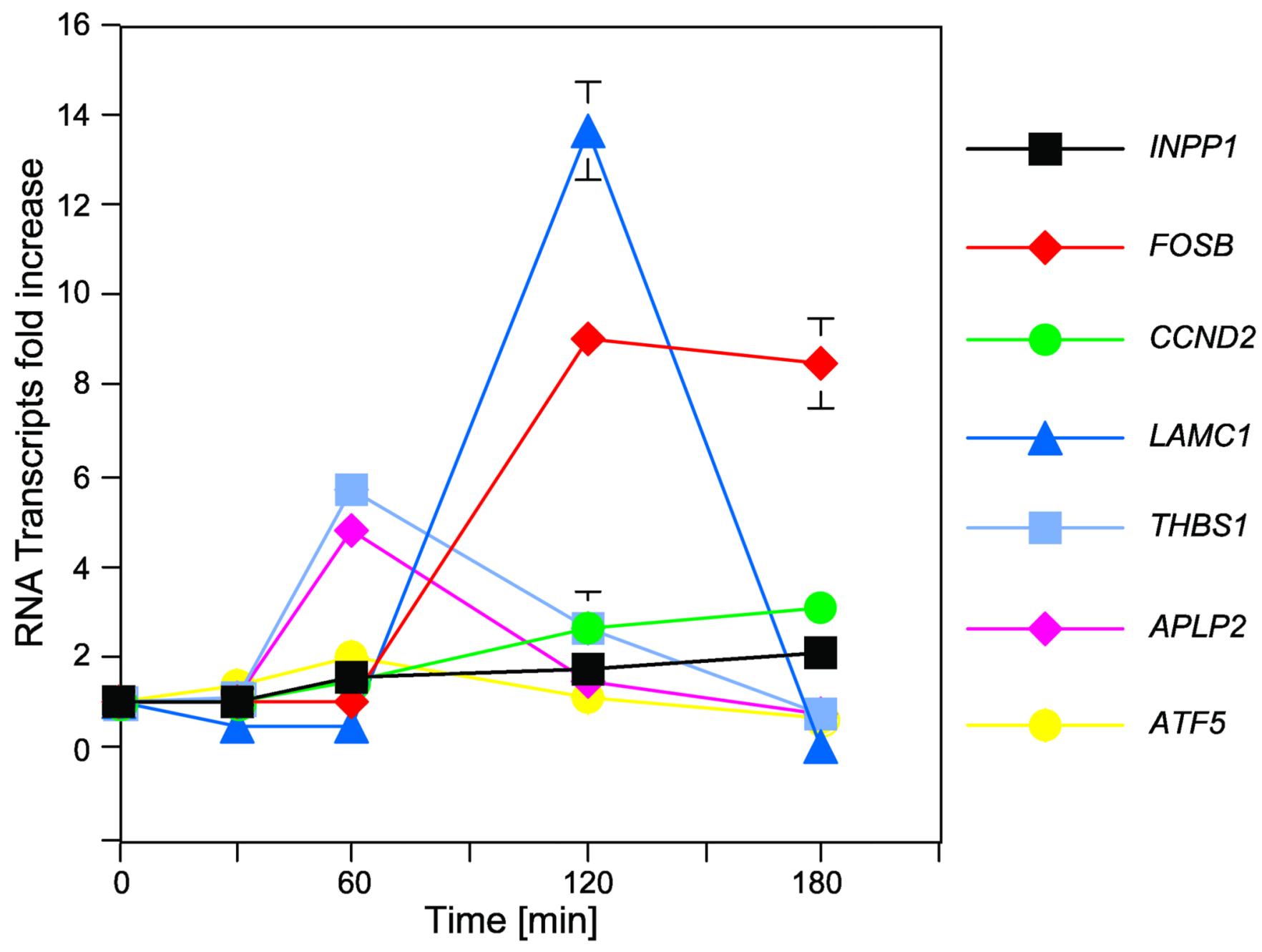

Fig. 1. Kinetics of gene expression in HCASM cells induced by early T. cruzi infection as evaluated by RT-PCR 


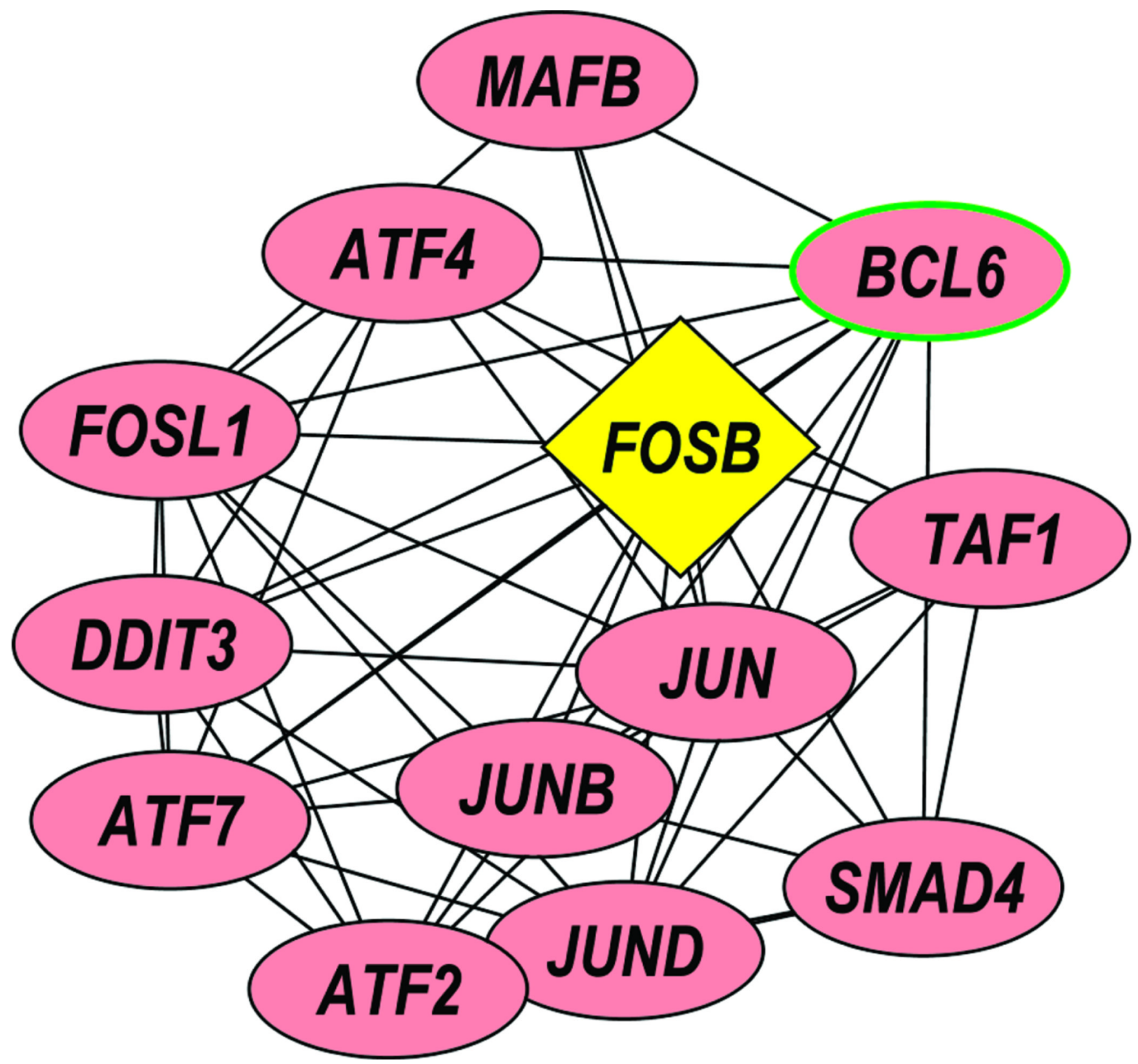

Fig. 2. FOSB Sub-network interaction

Initial nodes were populated with RT-PCR verified transcripts from gene transcription microarray data (yellow diamond nodes). Expansion nodes (pink ovals) were found to one degree of molecular interactions using the Michigan Molecular Interactions (MiMI) database. 


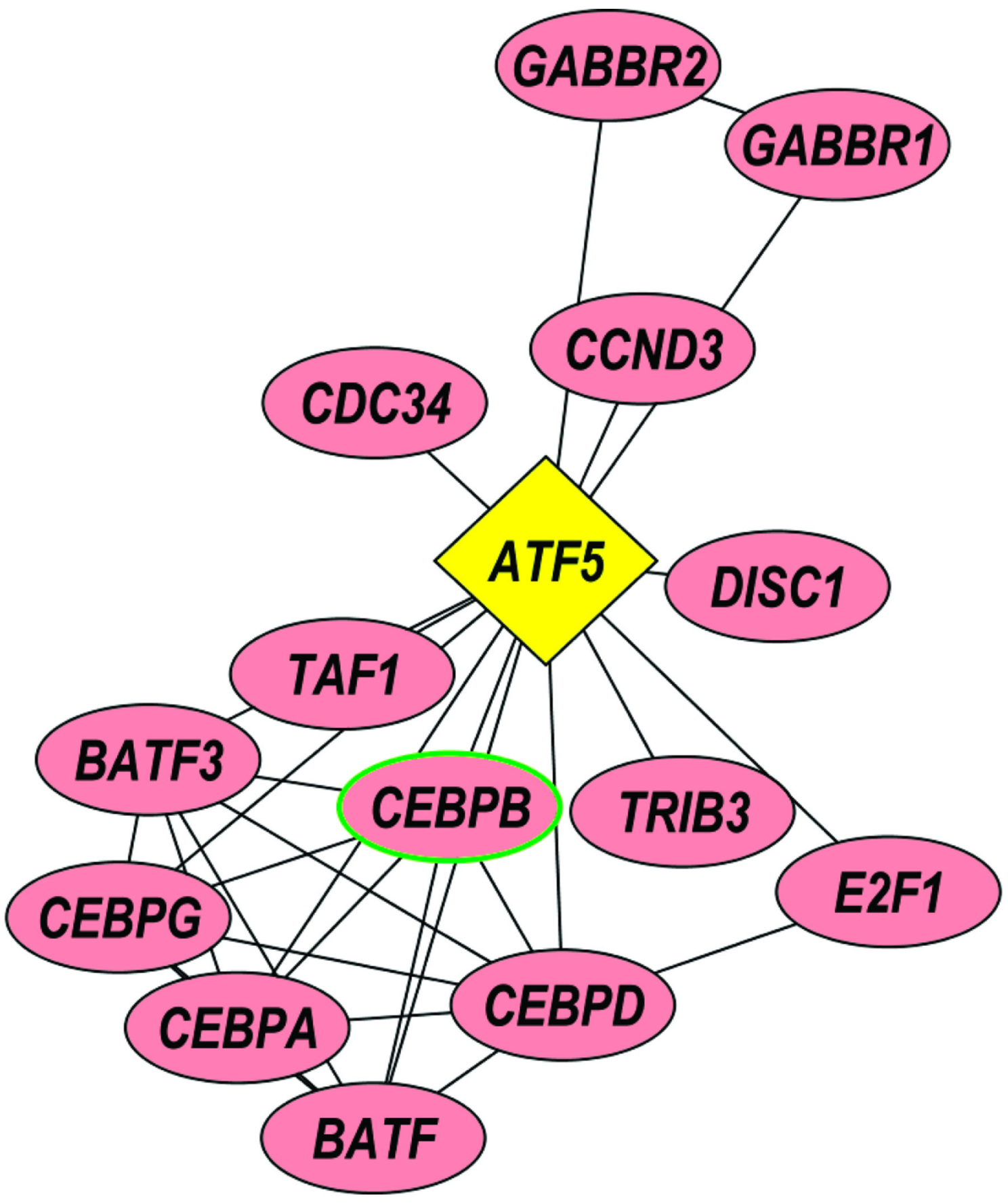

Fig. 3. ATF5 Sub-network interaction

Initial nodes were populated with RT-PCR verified transcripts from gene transcription microarray data (yellow diamond nodes). Expansion nodes (pink ovals) were found to one degree of molecular interactions using the MiMI database. 


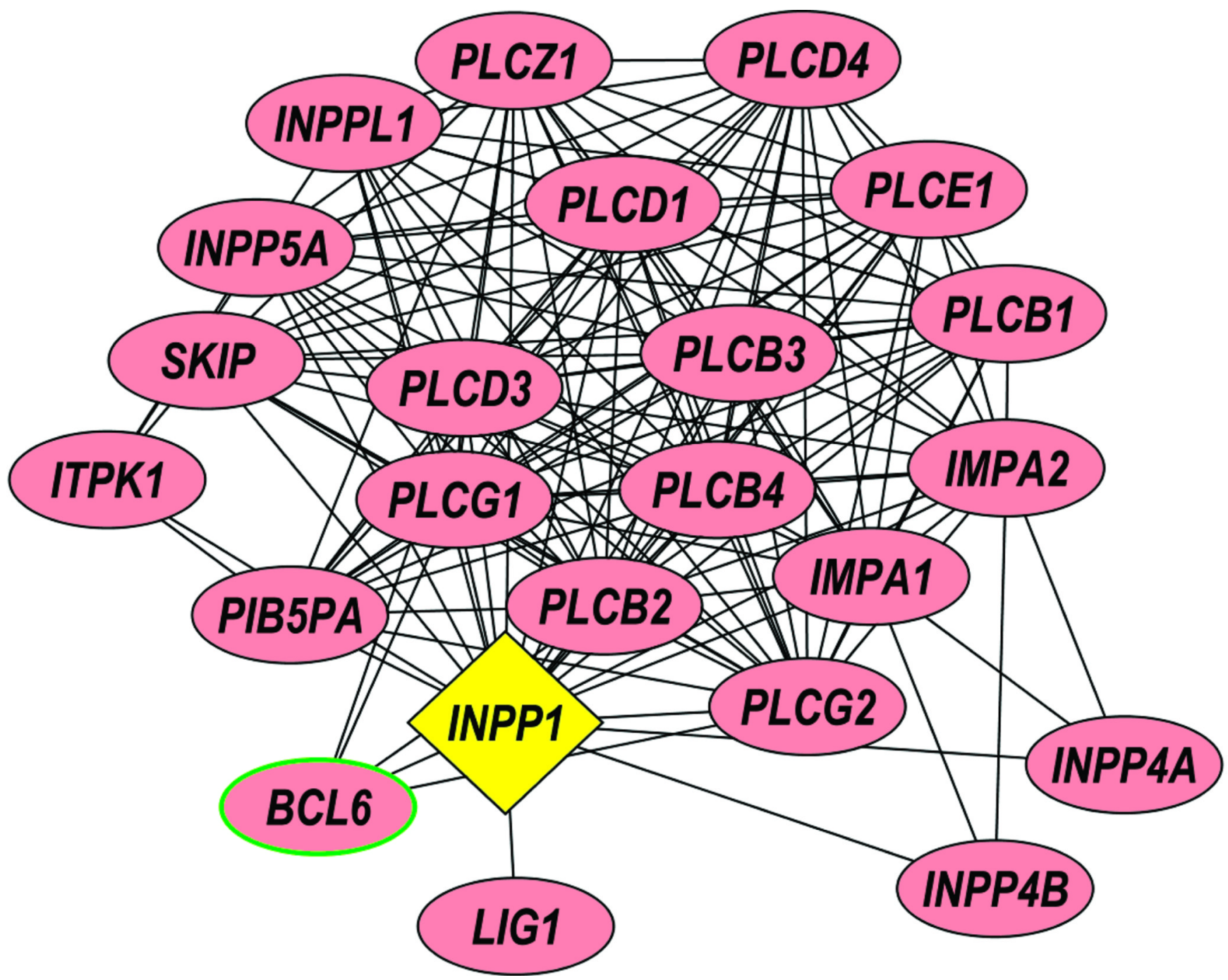

Fig. 4. INPPI Sub-network interaction

Initial nodes were populated with RT-PCR verified transcripts from gene transcription microarray data (yellow diamond nodes). Expansion nodes (pink ovals) were found to one degree of molecular interactions using the MiMI database. 


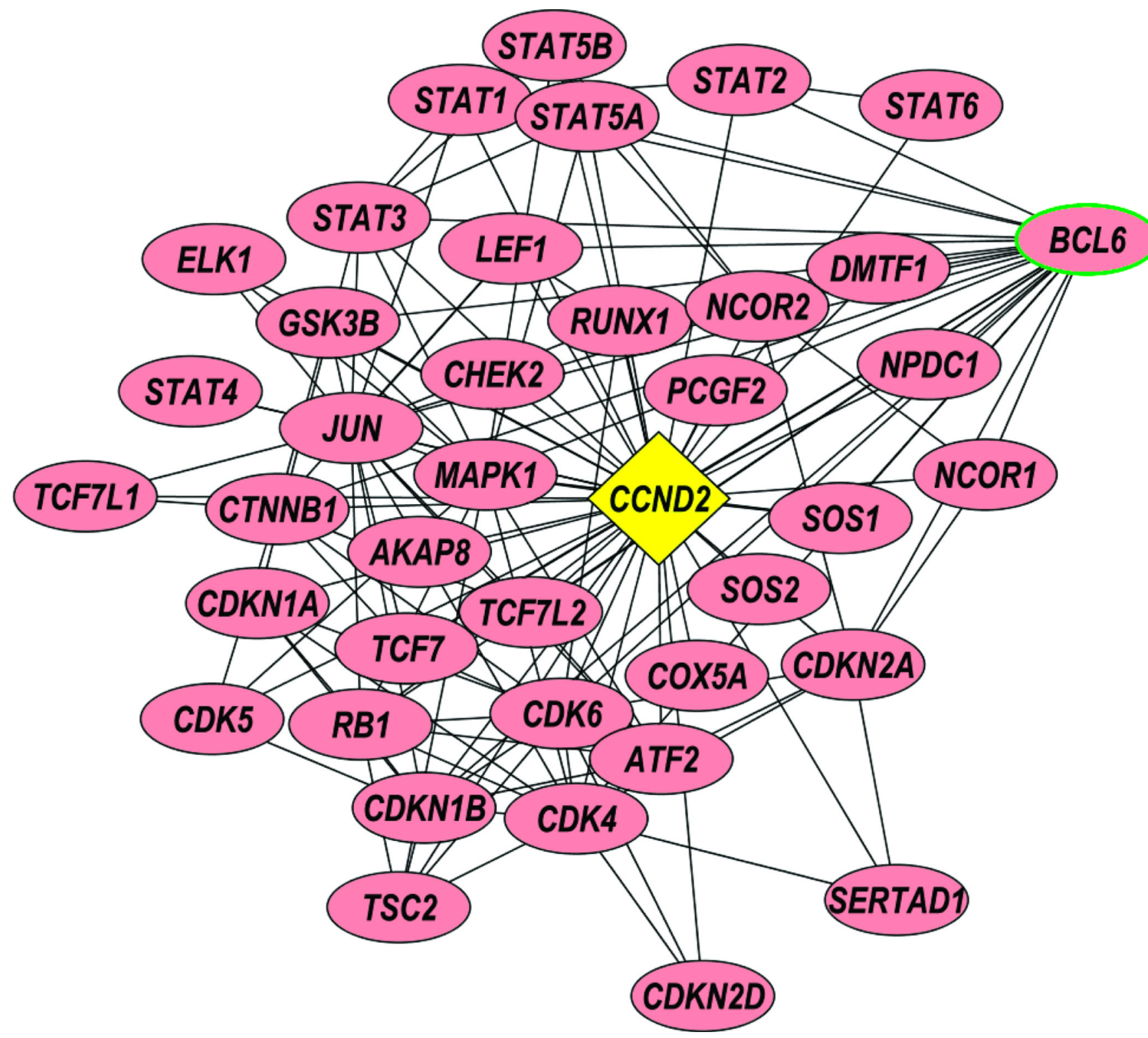

Fig. 5. CCND2 Sub-network interaction

Initial nodes were populated with RT-PCR verified transcripts from gene transcription microarray data (yellow diamond nodes). Expansion nodes (pink ovals) were found to one degree of molecular interactions using the MiMI database. 


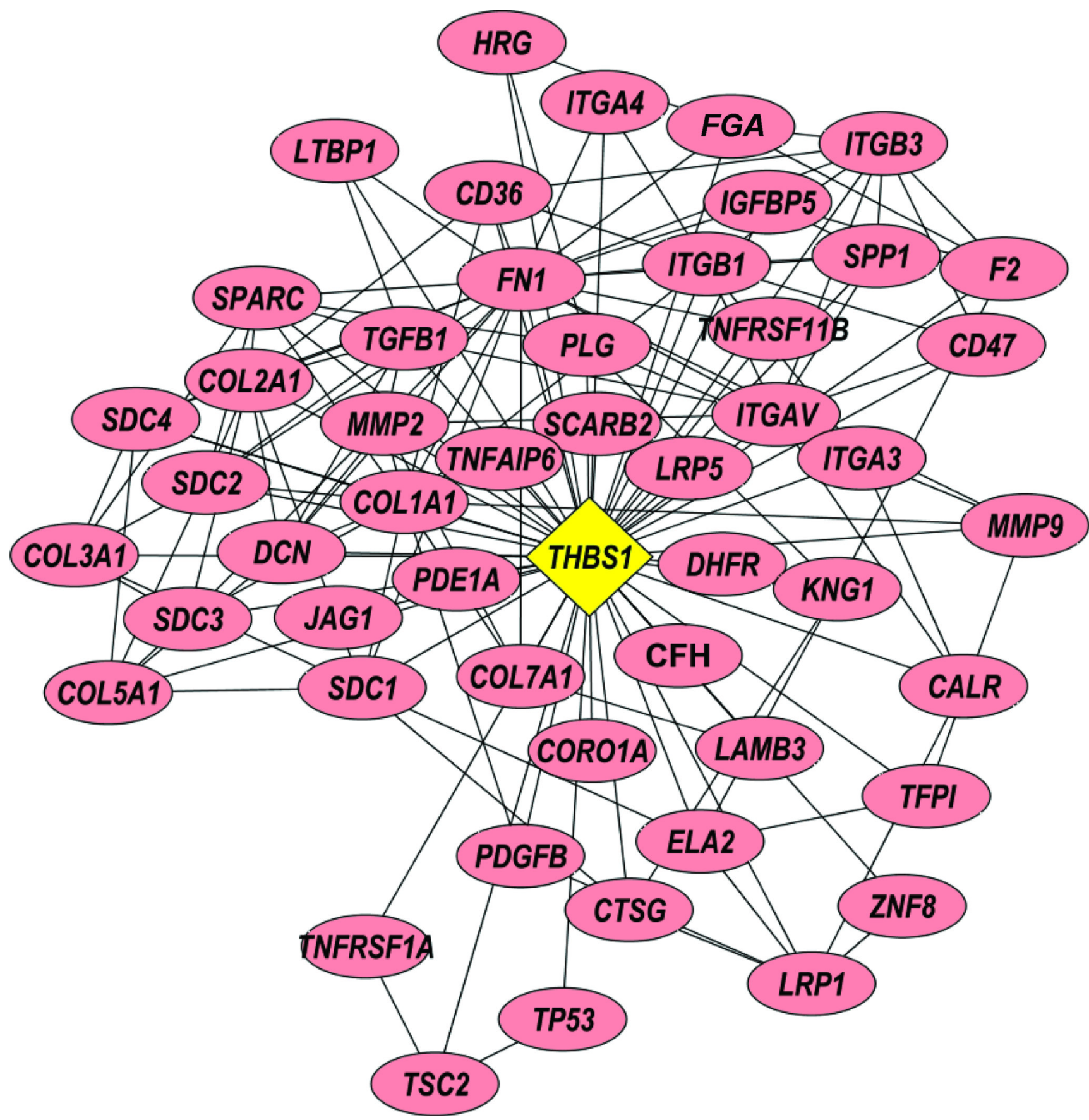

Fig. 6. THBS1 Sub-network interaction

Initial nodes were populated with RT-PCR verified transcripts from gene transcription microarray data (yellow diamond nodes). Expansion nodes (pink ovals) were found to one degree of molecular interactions using the MiMI database. 


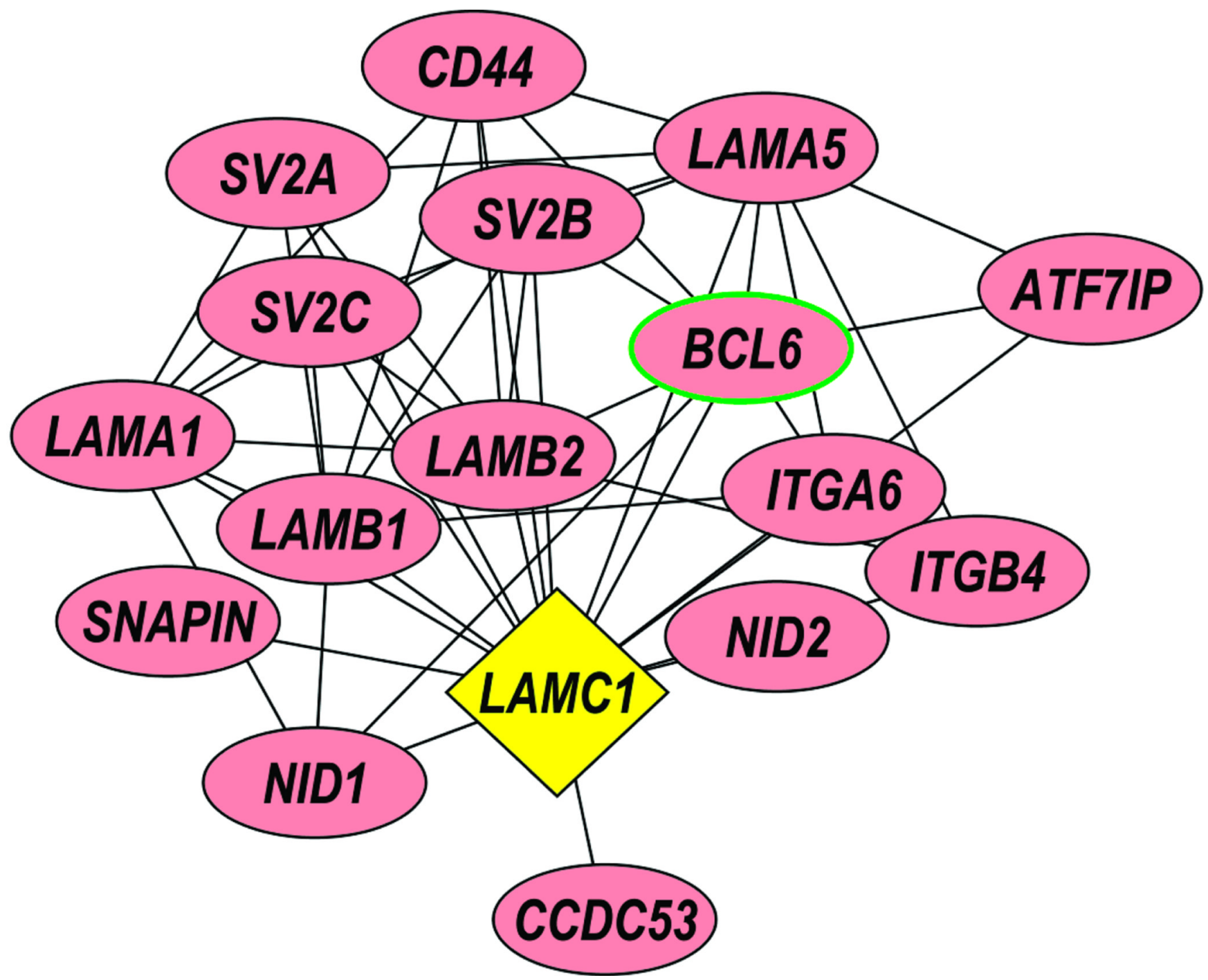

Fig. 7. LAMC1 Sub-network interaction

Initial nodes were populated with RT-PCR verified transcripts from gene transcription microarray data (yellow diamond nodes). Expansion nodes (pink ovals) were found to one degree of molecular interactions using the MiMI database. 


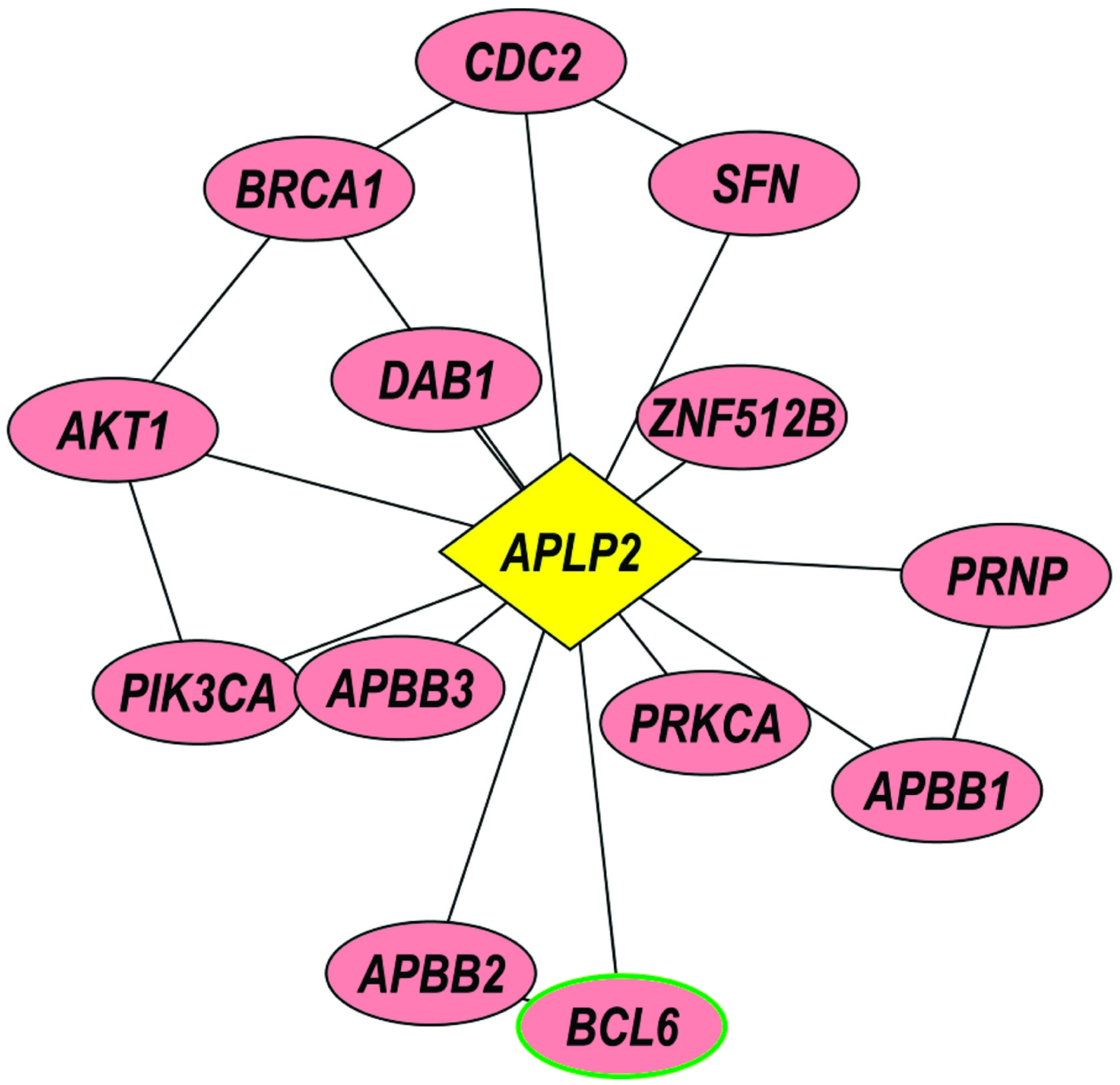

Fig. 8. APLP2 Sub-network interaction

Initial nodes were populated with RT-PCR verified transcripts from gene transcription microarray data (yellow diamond nodes). Expansion nodes (pink ovals) were found to one degree of molecular interactions using the MiMI database. 


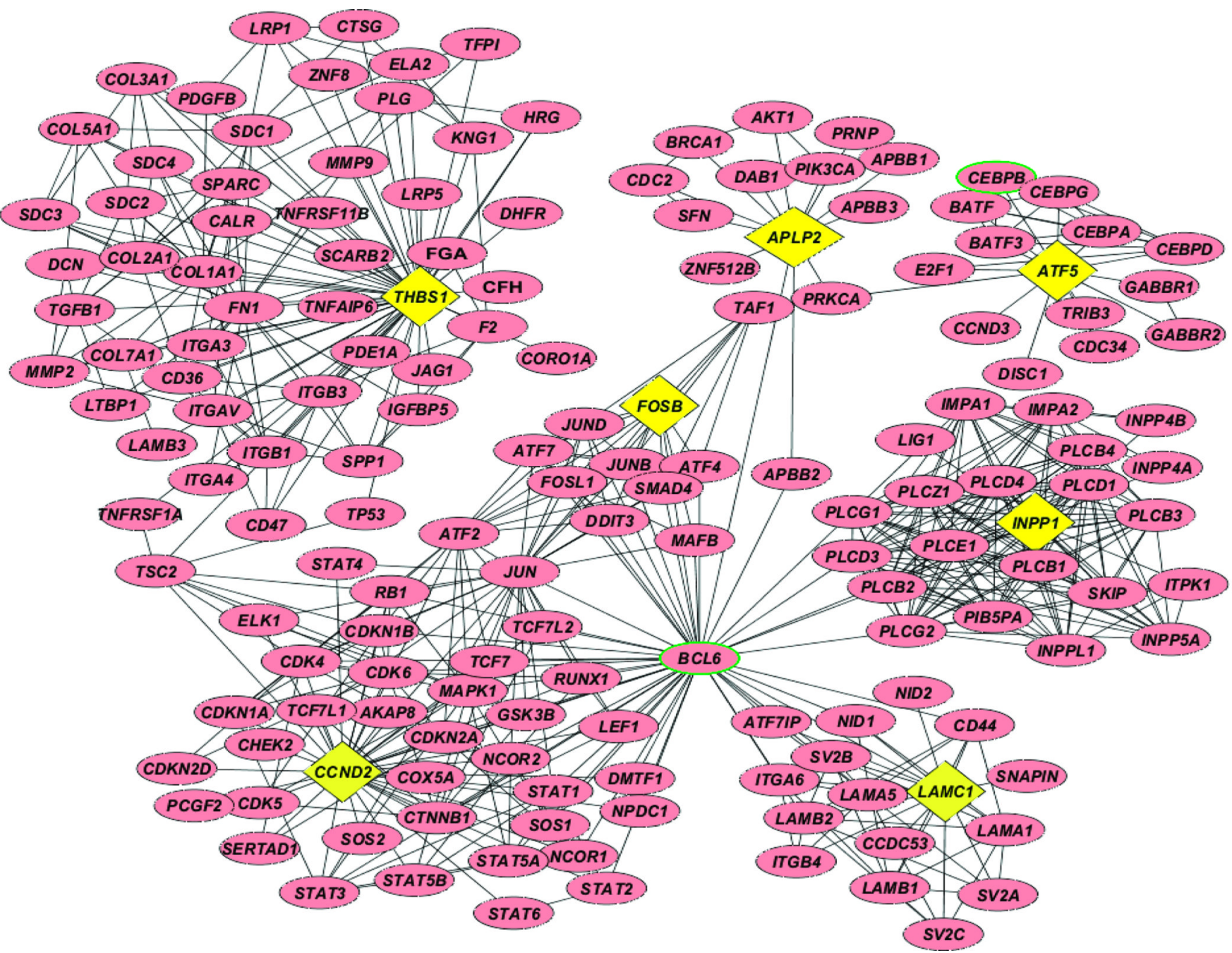

Fig. 9. Global interaction network of T. cruzi infection of HCASM cells Initial nodes were populated with RT-PCR verified transcripts from gene transcription microarray data (yellow diamond nodes). Expansion nodes (pink ovals) were found to one degree of molecular interactions using the MiMI database. The final interaction network was constructed by merging all seven initial seed node sub-networks. 
a)

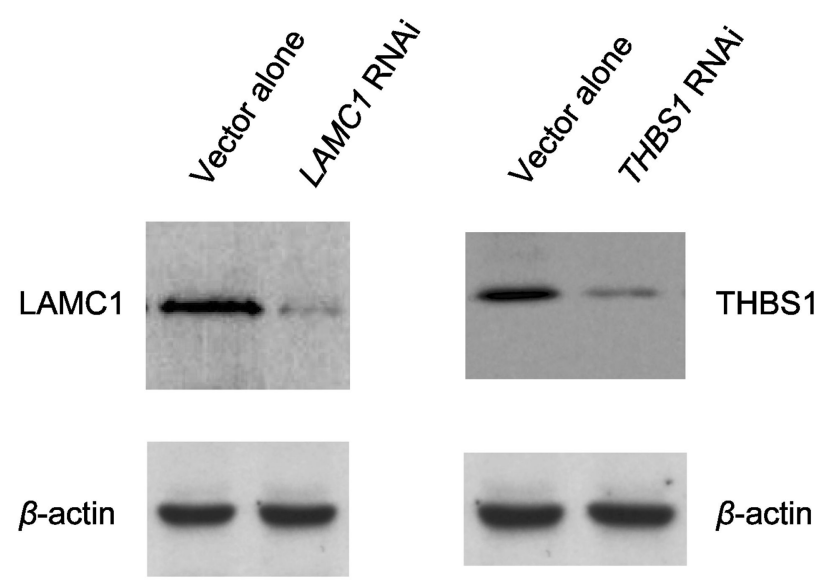

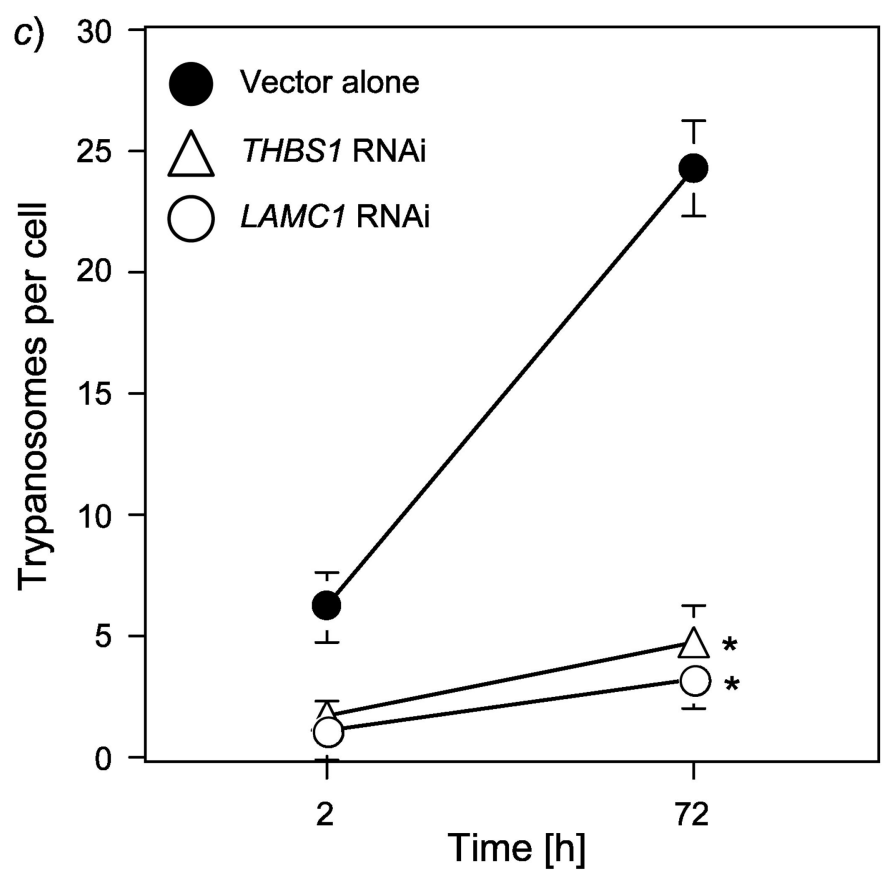

Fig. 10. Silencing the expression of $L A M C 1$ and $T H B S 1$ significantly reduces $T$. cruzi infection a) Immunoblot showing that RNAi of $L A M C 1$ reduces the expression in $L A M C 1$ in HeLa cells. b) Immunoblot showing that RNAi of THBS1 reduce the expression of THBS1 in HeLa cells. $c$ ) Kinetics of $T$. cruzi infection in HeLa cells in which LAMC1 and THBS1 were silenced by RNAi; * = significant difference compared to control value, cells transfected with vector alone $(p<0.05)$ by the Student's $t$ test. 


\section{Table}

Primer Sequences and Annealing Temperature Used for the PCR

\begin{tabular}{lllll}
\hline Gene Name & Accession No. & Forward Primer $\left(\mathbf{5}^{\prime} \rightarrow \mathbf{3}^{\prime}\right)$ & Reverse Primer $\left(\mathbf{5}^{\prime} \rightarrow \mathbf{3}^{\prime}\right)$ & $\boldsymbol{T}_{\mathbf{m}}\left[^{\circ} \mathbf{l}\right.$ \\
\hline LAMC1 & J03202 & CTCCATCAACCTCACGCTG & CGGCTGGTGTGGAACTTG & 56 \\
THBS1 & C52117 & CCCGTGGTCATCTTGTTCTGT & TTTCTTGCAGGCTTTGGTCTCC & 56 \\
FOSB & T61948 & TCATTGAATTGTGGTTGGCAGGAG & GGTGCGAGTGTGAGAGTGTGAG & 60 \\
ATF5 & T90841 & CCACCTTCCACCTGCCCTTAC & GCTGGGATGGCTCGTAGACTATG & 60 \\
CCND2 & H84154 & CGTCTGTGTTGGTGATCTTAGCG & CACTTGTGATGCCCTGACTGAG & 60 \\
INPP1 & H52141 & CCAGAGGGTGGGTGCCAATTC & GCAATGTTAGCAGCCTTCTCAGAG & 60 \\
APLP2 & H 89517 & CTTTGGTTCCTCCTTTCTTCAATCAC & GGCGTTATGTCCGTGCTGAG & 58 \\
Human 18s & M10098 & CGGACAGGATTGACAGATTGATAGC & TGCCAGAGTCTCGTTCGTATCG & 60 \\
\hline
\end{tabular}

